\title{
Lexical processing depends on sublexical processing: Evidence from the visual world paradigm and aphasia
}

\author{
Heather R. Dial ${ }^{1} \cdot$ Bob McMurray $^{2} \cdot$ Randi C. Martin ${ }^{3}$ \\ Published online: 3 April 2019 \\ (C) The Psychonomic Society, Inc. 2019
}

\begin{abstract}
Some early studies of people with aphasia reported strikingly better performance on lexical than on sublexical speech perception tasks. These findings challenged the claim that lexical processing depends on sublexical processing and suggested that acoustic information could be mapped directly to lexical representations. However, Dial and Martin (Neuropsychologia 96: 192-212, 2017) argued that these studies failed to match the discriminability of targets and distractors for the sublexical and lexical stimuli and showed that when using closely matched tasks with natural speech tokens, no patient performed substantially better at the lexical than at the sublexical processing task. In the current study, we sought to provide converging evidence for the dependence of lexical on sublexical processing by examining the perception of synthetic speech stimuli varied on a voice-onset time continuum using eye-tracking methodology, which is sensitive to online speech perception processes. Eight individuals with aphasia and ten age-matched controls completed two visual world paradigm tasks: phoneme (sublexical) and word (lexical) identification. For both identification and eye-movement data, strong correlations were observed between the sublexical and lexical tasks. Critically, no patient within the control range on the lexical task was impaired on the sublexical task. Overall, the current study supports the claim that lexical processing depends on sublexical processing. Implications for inferring deficits in people with aphasia and the use of sublexical tasks to assess sublexical processing are also discussed.
\end{abstract}

Keywords Speech perception $\cdot$ Perceptual categorization and identification $\cdot$ Eye movements $\cdot$ Cognitive

\section{Introduction}

A fundamental process in speech perception is translating continuous acoustic cues into abstract representational units

Electronic supplementary material The online version of this article (https://doi.org/10.3758/s13414-019-01718-3) contains supplementary material, which is available to authorized users.

Heather R. Dial

heather.raye.dial@gmail.com

1 Department of Communication Sciences and Disorders, University of Texas at Austin, 2504A Whitis Avenue (A1100), 4th Floor CMA, Austin, TX 78712-0114, USA

2 Department of Psychological and Brain Sciences, Department of Communication Sciences and Disorders, Department of Linguistics, Department of Otolaryngology, University of Iowa, Iowa City, IA, USA

3 Department of Psychological Sciences, Rice University, Houston, TX, USA that allow access to the meaning of the utterance. A common theoretical approach seen across many models of speech perception posits interactions among three levels of processing: (1) mapping acoustic cues onto abstract, speech-specific sublexical representations (e.g., syllables, phonemes), (2) mapping these representations onto lexical representations, and (3) mapping lexical representations onto semantic representations (e.g., Chen \& Mirman, 2012; Hickok \& Poeppel, 2007; Luce \& Pisoni, 1998; McClelland \& Elman, 1986; McQueen \& Cutler, 2000; Norris, 1994). An alternative approach posits only two of these stages: (1) mapping of acoustic information to lexical representations, and (2) mapping lexical representations onto semantic representations (e.g., Goldinger, 1998; Hickok \& Poeppel, 2000' Johnson, 1997;

\footnotetext{
${ }^{1}$ Note that the precursors to lexical-level processing are not well specified in Hickok and Poeppel's dual route model and may be (1) abstract and speechspecific (e.g., Hickok \& Poeppel, 2007, Fig. 2; see also Hickok, 2014), (2) based in acoustic space (e.g., Hickok \& Poeppel, 2000/2004, "sound-based representations of speech"), or (3) acoustic-phonetic (e.g., Hickok \& Poeppel, 2004).
} 
Pierrehumbert, 2001). These two classes of models contrast critically on the role, if any, of abstract, speech-specific sublexical representations in speech perception.

An important source of evidence for the claim that sublexical representations are not a causal part of the speech perception process comes from impaired populations. Here, a double dissociation has been observed between performance on tasks tapping sublexical processing and tasks tapping lexical processing (Basso, Casati, \& Vignolo, 1977; Blumstein, Baker, \& Goodglass, 1977a; Blumstein, Cooper, Zurif, \& Caramazza, 1977b; Miceli, Gainotti, Caltagirone, \& Masullo, 1980). The logic is that, if performance can be better on tasks assessing lexical processing than those assessing sublexical processing, then lexical processing must be independent of sublexical processing (implying direct mapping of auditory information to the lexical level).

However, recent work by Dial and Martin (2017) suggests that the findings from these studies reflect task artifacts and a failure to match stimulus discriminability between sublexical and lexical tasks rather than a true double dissociation. That study examined patterns of performance across an array of carefully designed tasks (syllable and word discrimination, auditory lexical decision, pictureword matching and auditory-written syllable matching) and stimuli (non-matching trials differed by a single distinctive feature of a single phoneme). Of note, two pairs of tasks were directly matched in terms of task demands and stimulus discriminability, such that the only difference was the lexical status of the stimuli (i.e., syllable vs. word discrimination, and syllable vs. picture matching). By matching task demands and stimulus discriminability, they were able to examine the relative contributions of sublexical and lexical processing to speech perception.

Dial and Martin (2017) found that accuracy on tasks tapping sublexical processing predicted accuracy on tasks tapping lexical processing. Critically, 11 of the 13 individuals with aphasia had either statistically equivalent performance on tasks tapping sublexical and lexical processing or performed better on tasks tapping sublexical processing. The only exceptions were two individuals who showed better performance on one lexical task relative to its sublexical counterpart, but not on other lexicalsublexical task comparisons. Overall, these findings support the claim that lexical processing depends on sublexical processing.

However, this finding may be premature for two reasons. First, the data were collected only for naturally produced speech. Consequently, these likely had prototypical values for phonetic cues like voice onset time (VOT), formant frequencies, etc. In contrast, a long history of work in speech perception suggests the largest differences are observed when examining how listeners respond to an entire continua of such cues, or by examining ambiguous regions (e.g., VOTs between $/ \mathrm{b} /$ and $/ \mathrm{p} /$ ). Thus, it is possible that greater differences between sublexical and lexical processes may be observed in a paradigm that offers a more finegrained analysis of the effect of cues like VOT.

Indeed, when prior studies have used entire continua, listeners with aphasia show abnormal identification functions for VOT (e.g., Blumstein et al., 1977b). Others have examined the influence of lexical factors on phoneme category boundaries (Blumstein, Burton, Baum, Waldstein, \& Katz, 1994). However, these studies have not directly compared an influence of VOT variation on sublexical versus lexical identification for the same set of participants. Thus, in the current study we manipulated a set of VOT continua and compared performance on a task tapping sublexical processing to one tapping lexical processing in a set of individuals with aphasia. As in Dial and Martin (2017), we matched task demands and stimulus discriminability between the two tasks to allow us to examine the relative contributions of sublexical and lexical processing on task performance.

Second, all of the data from Dial and Martin (2017) were collected using standard "off-line" tasks, which measure performance after perceptual processing is complete. A more online measure may provide a different view by examining the preliminary states of the decision before the final response. ${ }^{2}$ The outcomes of perceptual processing and decision-making processes do not always pattern together (cf., McMurray et al., 2018), and it is possible, for example, that early decision states reflect a dissociation between sublexical and lexical processing that are later merged in the final response.

These online processes can be assessed with the Visual World Paradigm (VWP). In the VWP, participants hear an auditory stimulus while viewing an array of potential visual responses. To locate the target response, participants must make one or more eye-movements, which reflect the degree to which each response is under consideration. Moreover, since participants make three to four eye movements over the course of a trial, the temporal dynamics of these eye movements can capture the unfolding dynamics of the decision. As a result, eye movements in the VWP have been shown to be extremely sensitive to real-time unfolding of speech perception and word recognition processes (e.g., see Allopena, Magnuson, \& Tanenhaus, 1998; Dahan, Magnuson, Tanenhaus, \& Hogan, 2001; Huettig \& Altmann, 2005; McMurray, Tanenhaus, \& Aslin, 2002; McMurray, Aslin,

\footnotetext{
${ }^{2}$ A related concern is that identification data fail to provide information regarding the extent to which competitor responses are considered before a final decision is made.
} 
Tanenhaus, Spivey, \& Subik, 2008; Salverda, Dahan, \& McQueen, 2003).

For example, McMurray and colleagues (McMurray et al., 2002) used the VWP to examine the sensitivity of lexical processing to fine-grained differences within a phonemic category. VOT was varied along a $/ \mathrm{b} /-/ \mathrm{p} /$ continuum spanning two words (e.g., bear/pear) and participants selected the picture (e.g., bear, pear) that matched their auditory percept. Identification responses reflected what is typically seen in categorical perception studies (e.g., Liberman, Harris, Hoffman, \& Griffith, 1957; for review see Diehl, Lotto, \& Holt, 2004): relatively consistent within-category responses around the endpoints of the continuum with a relatively sharp transition in responses across the category boundary. Eye movements to the competitor response, however, revealed a different pattern.

In order to isolate sensitivity to within-category detail, fixations to the competitor stimulus were analyzed after taking into account the response on that trial and the participant's own category boundary. McMurray et al. (2002) observed a graded pattern in the proportion of looks to the competitor, such that competitor fixations linearly increased as VOT approached the category boundary. Thus, eye movements suggested that acoustic-phonetic differences in VOT influence lexical level processing, even within a category. This graded pattern in the proportion of competitor fixations differs from the relatively sharp between-category transition observed in the identification task. Thus, online measures may reveal patterns of language processing not always observed in the final responses.

McMurray et al. (2002) suggest that within-category acoustic-phonetic differences are maintained at the lexical level. With respect to our primary question - the relation between sublexical and lexical processes - this could be interpreted in two ways. On the one hand, these findings could indicate that there is no need for an intermediate, abstract, speech-specific level; the fact that gradiency is maintained suggests that the system does not make a discrete category judgment, implying that acoustic-phonetic information maps directly onto lexical representations. Alternatively, these findings could indicate that gradiency in the acoustic-phonetic information is maintained across an abstract, speech-specific, sublexical level (e.g., Miller \& Volaitis, 1989; Oden \& Massaro, 1978); however, this sublexical level is gradiently sensitive to variation in the signal and continuously cascades into a lexical level. In this case, differences in the acoustic-phonetic information could lead to partial activation for multiple sublexical units (both $/ \mathrm{b} /$ and $/ \mathrm{p} /$ ). These partially active sublexical units would then map to the lexical level, leading to gradations of activity across lexical representations (e.g., across beach and peach). Indeed, supporting this, McMurray et al. (2008) used similar stimuli in an array of tasks including phoneme decision tasks. Across virtually all tasks, gradiency was observed, consistent with the idea of gradiency cascading from sublexical to lexical processing.

\section{Current study}

The current study instantiated phoneme identification (sublexical) and word-identification (lexical) tasks in the VWP to examine the relation between sublexical and lexical processing in people with aphasia (PWA). The benefit of using these tasks in the VWP is twofold. First, discrimination tasks, like those used in Dial and Martin (2017), unnecessarily tax short-term memory processes (e.g., Hickok \& Poeppel, 2004, 2007), which is potentially problematic in aphasia. The current tasks minimize short-term memory demands by utilizing a single auditory stimulus in each trial, along with a fairly natural labeling response. Second, the application of eye tracking to these rather canonical tasks may provide a more fine-grained measure of online speech perception.

We specifically sought to determine whether analyses of this more fine-grained speech perception measure (behavioral data collected across a VOT continuum) and data collected during the speech perception process (eye movements) would provide converging evidence with the measures reported in Dial and Martin (2017). To this end, we examined each PWA's performance on the phoneme and lexical identification tasks relative to a demographically matched control group. We operationally defined impaired performance as a deviation from the control group performance (i.e., a flattening of the [typically] sigmoidal response function for the mouse clicking, and an increase in the proportion of fixations to competitors).

We hypothesized that if acoustic cues are mapped directly to lexical representations (e.g., Goldinger, 1998; Hickok \& Poeppel, 2000; Johnson, 1997; Pierrehumbert, 2001), then it should be possible to observe substantially better performance on the lexical than the sublexical task. On the other hand, if sublexical processing is a necessary prerequisite for lexical processing, then no individual should perform normally on the lexical task (word identification) while demonstrating impaired performance on the sublexical task (phoneme identification). It should be noted that a dissociation may be possible in the other direction, with individuals showing deficits restricted to the lexical level. That is, these individuals could have a disruption of lexical representations per se, but no disruption of sublexical representations. Such a dissociation would be consistent with a hierarchical process in which sublexical processing is an independent, but required, preliminary process to lexical processing.

\section{Methods}

\section{Participants}

PWAs and healthy, age-matched controls were recruited from existing participant databases in the Brain and Language 
laboratory at Rice University. Informed consent was obtained in accordance with the Rice University Institutional Review Board regulations. Participants were compensated US $\$ 10$ per hour for participation.

Age-matched controls Eleven neurally healthy older adults were tested to obtain a baseline level of performance against which to compare PWAs. Their mean age was 71 years and they averaged 16.4 years of education. Following McMurray, Munson, and Tomblin (2014), one control participant was excluded from analyses because she had great difficulty performing the task $(<80 \%$ accuracy on endpoint stimuli), leaving ten control participants in subsequent analyses.

People with aphasia (PWA) Eight PWAs who were able to read written syllables and words participated. Note that five of the PWAs in the current study (QO, NMA, SH, MB, EV) were tested in Dial and Martin (2017), whereas three PWAs (CA, $\mathrm{HO}$, and NJ) were not tested in that study. The mean age of the PWAs was 62.5 years and the mean number of years of education was 15.4. Appendix A Table 5 contains detailed information about the PWAs, including aphasia type, lesion location, and performance on several neuropsychological tasks of language and memory.

Hearing All PWAs and seven of the control participants underwent an audiometry screening. Hearing thresholds were compared to international standards for median hearing thresholds as a function of age (International Organization of Standardization, 2000) by subtracting each hearing threshold from the age-adjusted medians at each frequency $(500,1,000$, 2,000, 3,000, and 4,000 Hz) and dividing by the normative median (e.g., Dalton, Cruickshanks, Klein, Klein, Wiley, \& Nondahl, 2003) to compute standardized (population relative ) z-scores. Positive $z$-scores reflect higher (poorer) hearing thresholds than would be expected for the person's age and the frequency of the tone. After averaging across the $z$-scores for the left and right ears to obtain a single hearing-loss estimate, we observed means that were relatively close between the PWAs and controls, with both groups having slightly positive means (controls: $M=0.38$, range $=-0.66-1.15$; PWAs: $M=0.56$, range $=$ 0.34-2.47). The one PWA who had a value greater than 2.0 standard deviations above the normative value $(\mathrm{HO})$ was a relatively young participant (57 years old) who had hearing that was worse than would be expected for her age, though the actual threshold (in $\mathrm{dB}$ ) for each tone was within the control range. ${ }^{3}$ Aside from $\mathrm{HO}$, the greatest hearing loss value for any PWA was 0.49 , clearly within the range of our controls. Additionally, for frequencies at or below $2,000 \mathrm{~Hz}$, only one PWA had clinically impaired hearing loss $-\mathrm{SH}$ - whose hearing screening was

\footnotetext{
${ }^{3}$ Perhaps more importantly, this PWA was within the control range on all dependent measures in the experimental tasks presented below.
}

conducted without hearing aids, though we note that he wore hearing aids during testing. Thus, hearing loss should not be an important contributing factor to performance in the following tasks.

\section{Apparatus}

All of the visual stimuli were presented on a Dell desktop computer (display resolution $1,920 \times 1,080$ ) running Experiment-Builder (SR Research Ltd). The auditory stimuli were presented via Mutant MIG-NC102 noise canceling headphones and adjusted to a comfortable listening level for each participant.

\section{Materials}

Phoneme identification Auditory stimuli for the phoneme identification task were synthetic $/ b a /-/ p a /$ stimuli that varied along a nine-step VOT continuum from 0 to $40 \mathrm{~ms}$ in 5 -ms increments (the original stimuli from McMurray et al., 2008). ${ }^{4}$ There were also two filler stimuli that were unrelated to the /ba/ and /pa/ stimuli (/la/ and /sha/). The visual stimuli were four written syllables that corresponded to the auditory stimuli (i.e., "BA," "PA," "LA," and "SHA"). Each letter was positioned in a single quadrant and was 631 pixels (width) $\times 422$ pixels (height). Quadrant-letter pairings and the location of the target relative to the competitor stimulus were pseudorandomized across blocks in order to account for effects of screen placement on looking times.

Lexical identification Lexical stimuli were six synthetic ninestep VOT continua $(0-40 \mathrm{~ms}$, in 5 -ms increments) spanning two words (bale/pail, beach/peach, butter/putter, bear/pear, bomb/palm and bump/pump), plus six/1/- initial (ladder, lamp, leg, lip, leaf and lock) and six /sh/- initial word stimuli (shoe, shark, sheep, shell, ship and shirt) as filler items (from McMurray et al., 2002). All visual stimuli were colored line drawings developed by the McMurray lab using a standard lab protocol (McMurray, Samuelson, Lee, \& Tomblin, 2010). Quadrant-picture pairings and the location of the target relative to the competitor stimulus were pseudo-randomized across blocks as in the phoneme identification task.

\section{Procedure}

Each testing session began with calibration and validation of the eye tracker. Participants were then auditorily and visually presented with the instructions for the task, with the

\footnotetext{
${ }^{4}$ We had some concerns that PWA might struggle with the synthetic stimuli. However, identification accuracy for stimuli at the endpoints of the synthetic VOT continuum was closely related to performance on a similar minimal pairs task using natural speech tokens.
} 
experimenter answering questions as needed. Both the phoneme and lexical identification tasks started with brief training before participants underwent testing. Each participant participated in a single session for phoneme identification and two sessions for lexical identification, with each session conducted on a different day. The lexical task was modeled closely after McMurray et al. (2002), and the phoneme task after McMurray et al. (2008).

Phoneme identification To ensure that each participant could accurately identify the orthographic responses used in the experiment, they were asked to read each written syllable aloud. They were corrected by the experimenter if necessary and then asked to read the syllables aloud without assistance. All PWAs and controls were able to read the syllables aloud without the experimenter's assistance during the first or second presentation of the written stimuli.

Next, participants completed 12 practice trials. On these trials, the four orthographic responses were presented along with a blue circle at screen center (Fig. 1). After $500 \mathrm{~ms}$, the circle turned red. Participants clicked on the red circle to trigger presentation of the auditory stimulus, ensuring that the participant's gaze was focused on the center of the screen at the start of each trial. An auditory stimulus was presented and the participant clicked on the orthographic response. The experimenter corrected any errors.

Experimental trials were presented in the same way as the practice trials, but without experimenter feedback. Participants were encouraged to respond at a pace that felt natural to them, though they were also informed that if they did not respond within $3 \mathrm{~s}$, the task would automatically proceed to the next trial. Eighteen trials were presented per block, with a drift correction procedure administered between blocks. Participants were informed that the arrangement of the four syllables would change after each drift correction and that they should wait to click on the red circle until they had familiarized themselves with the current layout. Testing in the phoneme identification task was completed within a single session consisting of 24 blocks of 18 trials each.
Each of the nine VOT steps was presented 24 times and each of the two filler items was presented 108 times, for a total of 432 trials (as in McMurray et al., 2008). The session lasted approximately $45 \mathrm{~min}$.

Lexical identification The lexical identification task began with a training session to familiarize the participants with the picture responses. All 24 images were shown one at a time with their name printed below, which the experimenter read aloud. Next, participants completed a second training phase where they were presented with four pictures, one in each quadrant (Fig. 1), with a blue circle in the center of the screen that turned red after $500 \mathrm{~ms}$. After clicking on the red circle, a printed word appeared in its place and the participant clicked on the corresponding picture. Each of the 24 stimuli appeared twice (in orthographic form) during this training for a total of 48 trials. Neither controls nor PWAs made any errors during this training phase.

Finally, participants performed practice trials in which a spoken word was presented instead of a written word, with the endpoint stimuli from the lexical task presented for the $/ \mathrm{b} /$ and $/ \mathrm{p} /$ initial words. The experimenter corrected the participant if they made any errors. All PWAs and controls responded with at least $92 \%$ accuracy during this practice phase. Practice was completed before both lexical identification testing sessions. The format of the testing phase was identical to this final practice phase but with tokens from the entire continua and no feedback.

The lexical identification task included 60 blocks of 18 trials each. We chose to block trials by word pair as we believed that random presentation of the word pairs would increase the cognitive effort required for this task (a demand not present on the phoneme identification task). This could have differentially affected performance on the task, particularly for PWAs. Filler stimuli were paired with each continuum such that only one $/ 1 /$ and one $/ \mathrm{sh} /$ filler was used in a block. All blocks for a word pair were completed in succession before moving on to the next word pair. Participants were reminded that the position of

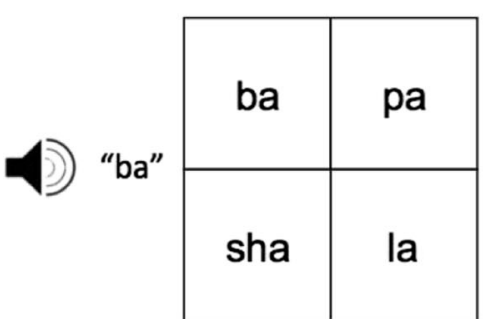

Fig. 1 Graphic depiction of the screen layout for the phoneme (left) and lexical (right) identification tasks. Dividing lines have been added for emphasizing the quadrants, but were not presented during the actual

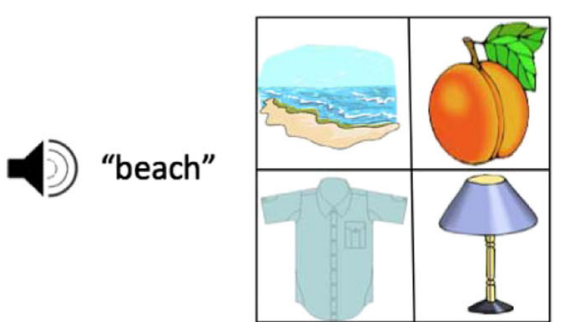

task. Note that the position of the written syllables and pictures relative to each other was counterbalanced 


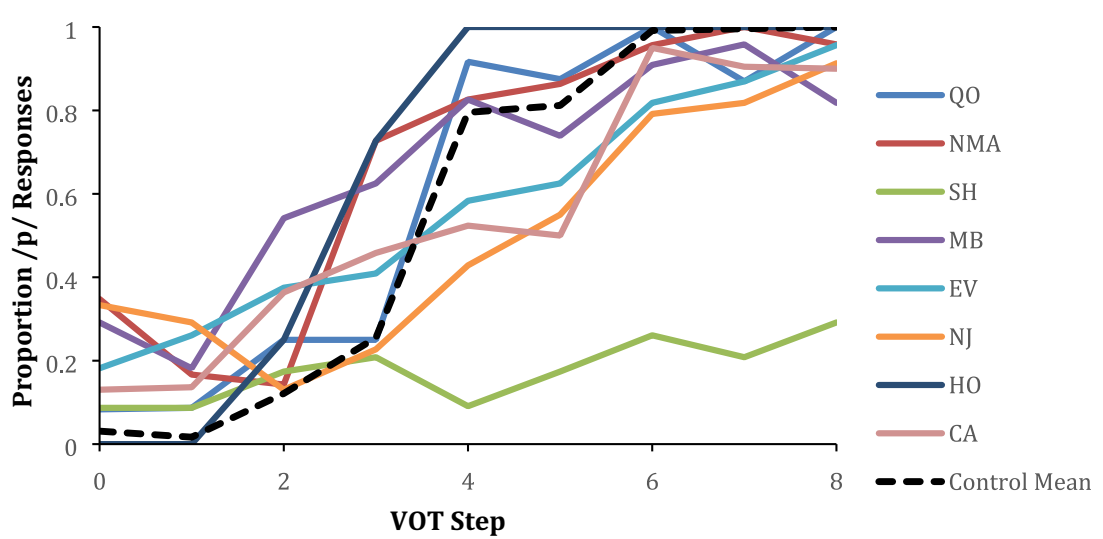

Fig. 2 Identification data for the phoneme identification task. Lines labeled with initials represent individual PWA

the pictures would change after each drift correction and that they should wait to click on the red circle until they had familiarized themselves with the current layout. Each of the nine VOTs for each of the six word pairs was presented ten times for a total of 540 experimental trials. There were also 540 filler trials (fillers words presented 45 times each). Due to the total number of trials $(1,080)$, the task was broken into two 1-h sessions, each containing 30 blocks (540 trials).

\section{Eye-tracking analysis}

Participants' eye movements were recorded using the tower mounted EyeLink 1000 Plus (SR Research Ltd) eye tracker, using a 500-Hz sampling rate. A standard 9-point calibration procedure was run at the beginning of the experiment, and a drift-correction procedure was run every 18 trials. Eye-movement data were automatically parsed into saccades, fixations, and blinks. These events were pre-processed with EyelinkAnal software (McMurray, 2017a). This combined adjacent saccades and fixations into a single "look," which began at saccade onset, lasted to the end of the fixation, and was directed to the mean point of gaze during the fixation. In assigning looks to regions of interest, the boundaries of the pictures were extended by 100 pixels to account for any noise in the eye movements. This did not result in any overlap of the areas of interest.

\section{Results/discussion}

We first analyzed the identification data (the mouseclick responses) as this was a prerequisite for analysis of the eye-movement data. Next, we analyzed the fixations. Raw data and $\mathrm{R}$ scripts have been posted to a public repository and are available at: https://osf.io/ $9 \mathrm{z} 8 \times 2$.

\section{Identification data}

Approach We excluded trials where a filler picture was chosen or where no response was made within 3,000 ms. PWAs chose a filler picture on $0-.01 \%$ of phoneme identification trials $(<.01 \%$ for controls $)$ and $0-.002 \%$ of lexical identification trials $(0 \%$ for controls). For PWAs, the number of trials that timed out ranged from $.69 \%$ to $5.32 \%$ of trials for phoneme identification $(0$ $2.31 \%$ for controls) and $.56 \%$ to $5.93 \%$ of trials for lexical identification $(0-1.94 \%$ for controls). Importantly, when examining both filler picture selections and trials that timed out, no individual had greater than $6 \%$ of trials excluded from analyses.

Phoneme and lexical identification data (Figs. 2 and 3) were fit using a four-parameter logistic function (Eq. 1). The goal of this was twofold. First, we used the parameters of these fits as dependent variables for analysis of the identification data. Second, these fits were required for our analysis of the fixation data, which were analyzed relative to each participant's boundary.

$P(/ p /)=\frac{\max -\min }{1+\exp \left(4 \times \frac{\text { slope }}{\max -\min } \times(\text { boundary-step })\right)+\min }$

The four-parameter logistic function models the proportion of $/ p /$ responding as a function of VOT step. Four parameters are used to describe each participant's responding: the maximum and minimum asymptotes ( $\max$ and $\min$ ), the category boundary (the step at which the function crosses 50\% of the distance between max and $\mathrm{min}$ ), and the slope of the function (the derivative at the boundary). In this function, the slope is classically seen as the degree to which categorization is discrete or gradient, and the asymptotes reflect the accuracy of categorization at the end-points of the continuum. These two parameters were expected to reflect differences in categorization by PWAs. The boundary reflects where along the VOT 


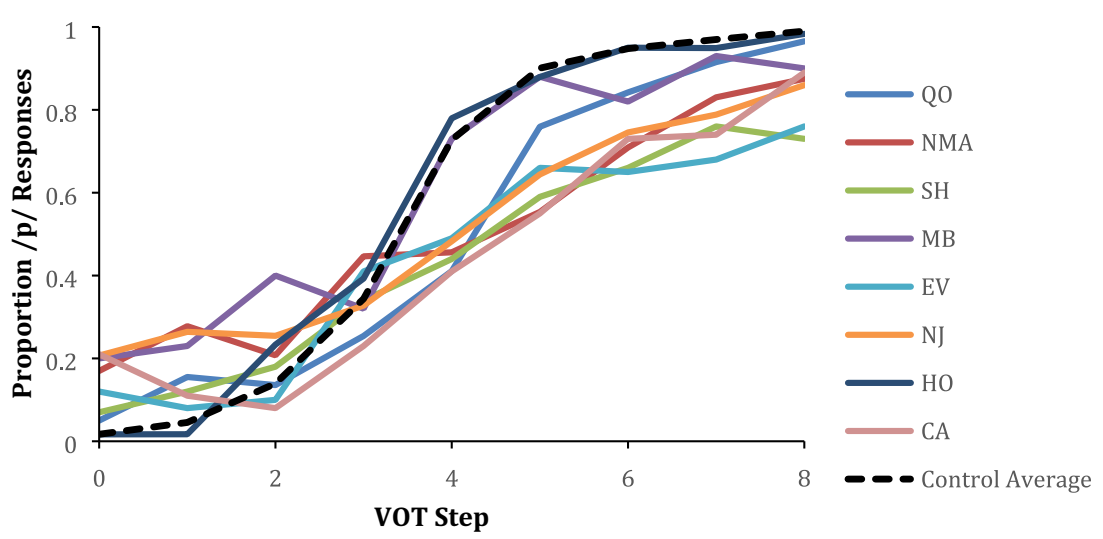

Fig. 3 Identification data for the lexical identification task, collapsed across word pairs with $R^{2}>.80$. Lines labeled with initials represent individual PWA

continuum the $/ \mathrm{b} /$ and $/ \mathrm{p} /$ category separates, and is not seen as a direct measure of the quality of categorization.

Identification data were fit using a constrained gradient descent algorithm that minimizes the least squared error while ensuring that the function remained between 0 and 1 . This algorithm (McMurray, 2017b) was implemented in MATLAB (MATLAB and the Optimization Toolbox, Release, 2015).

Each participant's data were fit separately in the phoneme identification condition. $R^{2}$ was used as a metric of goodness of fit to the data (Appendix B Table 6), with an adequate fit defined as $R^{2}>.80$. Data from all controls and seven of eight PWAs displayed adequate fits for the phoneme identification task. SH's phoneme identification data were flat (Fig. 2, light green), so the four-parameter logistic function, a sigmoidal function, was unable to provide an adequate fit.

For the lexical identification task, ${ }^{5}$ we began by attempting to fit individual data for each word pair for each participant (Supplementary Fig. S1.1). However, for the PWAs some word pairs were not successfully fit at .80 or higher because there was little or no difference between the asymptotes (Supplementary Fig. S1.1; CA: bale/pail $R^{2}=$ .04, bomb $/$ palm $R^{2}=.51 ; \mathrm{EV}:$ bale/pail $R^{2}=.51 ; \mathrm{MB}$ : bale/ pail $R^{2}=.61$, beach $/$ peach $R^{2}=.73 ; \mathrm{SH}$ : beach $/$ peach $R^{2}=$ .66, unable to fit bump/pump or bomb/palm). In order to minimize noise and maximize the number of trials included for each participant, we thus collapsed the data across word pairs, excluding these pairs from that participant's collapsed data. Appendix B Table 6 presents the $R^{2}$ for these

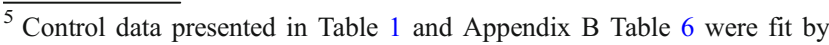
participant but collapsed across word pairs and participants for ease of presentation. Note, though, that we also looked at individual word pairs and participant data. Collapsing across participants while looking at individual word pairs, the logistic fit for each word pair ranged from .98 to 1 and the lowest $R^{2}$ for a word pair within a single control participant was .88 .
}

final fits and Fig. 3 presents the collapsed data on which these fits were based.

After ensuring adequate fit, we next examined the individual parameters as descriptors of the identification data, focusing on the asymptotes and slope. For each parameter, we compared (1) the PWA group to the control group using a $2 \times 2$ ANOVA; (2) each individual PWA to controls using Crawford and Howell's (1998) modified $t$-test and $z$-scores to determine levels of impairment; and (3) Pearson's correlation analysis to examine the relation between phoneme and lexical task dependent measures. If PWAs show relatively spared lexical phonological processing together with impaired sublexical phonological processing, then the two-way ANOVA should reveal an interaction such that the difference between performance on lexical and sublexical tasks was greater for the PWAs than for controls, with the interaction resulting from a greater reduction in sublexical performance for PWAs. However, even if no such interaction is obtained, it is important to examine individual PWA's performance, as impaired performance at the lexical level could be due to deficits other than impaired sublexical phonological processing - such as a disruption of lexical-phonological or lexical-semantic representations.

The crucial question in relation to our hypotheses is whether any individual PWA showed substantially better performance on the lexical task, which might be expected if lexical processing did not depend on sublexical processing. Thus, we examined the relative impairment of each PWA to determine if any showed substantially worse performance on the sublexical than the lexical task. Correlations were examined because if lexical processing deficits derive from sublexical processing deficits, then a correlation between the degree of deficit on the two measures should be observed. 
Table 1 Asymptotes and amplitudes for phoneme and lexical identification tasks for PWA and controls

\begin{tabular}{|c|c|c|c|c|c|c|}
\hline \multirow[t]{2}{*}{ PWAs } & \multicolumn{3}{|l|}{ Phoneme } & \multicolumn{3}{|l|}{ Lexical } \\
\hline & Minimum & Maximum & Amplitude & Minimum & Maximum & Amplitude \\
\hline QO & 0.14 & 0.94 & 0.80 & 0.09 & 0.95 & 0.86 \\
\hline NMA & 0.22 & 0.92 & 0.70 & 0.18 & 0.88 & 0.70 \\
\hline $\mathrm{SH}$ & 0.09 & 0.29 & 0.20 & 0.07 & 0.76 & 0.69 \\
\hline MB & 0.20 & 0.87 & 0.67 & 0.27 & 0.88 & 0.61 \\
\hline EV & 0.18 & 0.96 & 0.78 & 0.08 & 0.70 & 0.62 \\
\hline NJ & 0.24 & 0.89 & 0.65 & 0.21 & 0.85 & 0.64 \\
\hline $\mathrm{HO}$ & 0.00 & 1.00 & 1.00 & 0.02 & 0.97 & 0.95 \\
\hline $\mathrm{CA}$ & 0.13 & 0.95 & 0.82 & 0.12 & 0.86 & 0.74 \\
\hline Control mean (range) & $0.03(0.00-0.14)$ & $0.98(0.94-1.00)$ & $0.95(0.86-0.99)$ & $0.02(0.00-0.05)$ & $0.99(0.96-1.00)$ & $0.97(0.92-1.00)$ \\
\hline
\end{tabular}

Analysis of asymptotes The maximum and minimum asymptotes represent the proportion of trials on which a participant responded $/ \mathrm{b} /$ or $/ \mathrm{p} /$ at the endpoints of the VOT continuum. For analysis we converted this to amplitude, the difference between asymptotes (Table 1); here, an amplitude of 1 indicates perfect categorization, and 0 indicates a complete failure to separate the categories.

Amplitude was analyzed with a two-way ANOVA using task (phoneme vs. lexical) as a within-subjects factor and group (PWA vs. control) as a between-subjects variable. Results showed a highly significant main effect of group, $F$ $(1,32)=17.76, p<.001$, no main effect of task $(p=.74)$, and no interaction $(p=0.96)$. For both tasks, all but one PWA scored below the range of controls. Thus, as a group, the PWAs showed strikingly smaller amplitudes relative to controls and the discrepancy was of the same magnitude for the sublexical and lexical tasks.

To quantify an individual PWA's discrepancy from the control mean, we used the Crawford and Howell (1998) modified $t$-test (see Table 2). Seven of the eight PWAs were impaired relative to controls on phoneme identification ( $p$ 's from $<.001$ to .03 ). Importantly, these seven PWAs were also impaired on the lexical identification task ( $p$ 's $<0.001) .{ }^{6}$ To determine relative levels of impairment, we converted all PWA scores to $z$-scores relative to the control group (Table 2; see also Fig. 4b). The degree of

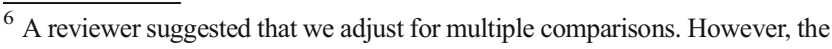
issue was not whether an individual PWA differed significantly from controls on a particular task, but whether performance was worse on the lexical than phoneme task. These comparisons could be made whether or not the PWA differed significantly from controls on one or both tasks. Nonetheless, we have provided the significance level for each task for each PWA using a false discovery rate correction in Supplement S2, Tables S2.1 and S2.3. The results for these two tasks are the same, with only $\mathrm{HO}$ failing to differ significantly from controls.
}

impairment, indexed by $z$-scores, was much larger for the lexical task than for the phoneme task for six of the seven PWAs. The exception was SH, whose $z$-score for the lexical task was smaller than the phoneme task (phoneme $z=-$ 15.61 vs. lexical $z=-10.81$ ).

$\mathrm{SH}$ was well outside of the normal range on the lexical task, as his performance was much worse than the healthy controls $(\sim 11$ standard deviations poorer than the controls). Further, interpretation of SH's data warrants caution, as the four-parameter logistic function was unable to fit the data for two of the word pairs, and had a poor fit for a third word pair. If we look at the data including the four word pairs that could be fit, the $z$-score for the phoneme task is less deviant than that for the lexical task (15.61 vs. -23.97 , respectively; see Supplement S2, Tables S2.2 and S2.4 for data including poorly fit word pairs). We also note that when looking solely at the stimuli that were most closely matched in terms of the coarticulatory influence of vowel on acoustic cues of the

Table 2 Results of Crawford and Howell's (1998) modified $t$-test and $z$ scores comparing PWA's amplitudes to controls

\begin{tabular}{|c|c|c|c|c|c|c|}
\hline \multirow[t]{2}{*}{ PWA } & \multicolumn{3}{|c|}{ Phoneme } & \multicolumn{3}{|l|}{ Lexical } \\
\hline & $t$ & $p$ & $z$ & $t$ & $p$ & $z$ \\
\hline QO & -3.00 & 0.01 & -3.15 & -4.13 & $<0.01$ & -4.33 \\
\hline NMA & -4.96 & $<0.001$ & -5.21 & -10.12 & $<0.001$ & -10.62 \\
\hline SH & -14.88 & $<0.001$ & -15.61 & -10.31 & $<0.001$ & -10.81 \\
\hline MB & -5.55 & $<0.001$ & -5.82 & -13.16 & $<0.001$ & -13.80 \\
\hline EV & -3.53 & 0.01 & -3.71 & -12.81 & $<0.001$ & -13.44 \\
\hline NJ & -6.08 & $<0.001$ & -6.38 & -12.17 & $<0.001$ & -12.76 \\
\hline $\mathrm{HO}$ & 0.95 & 0.37 & 1.00 & -0.74 & 0.48 & -0.77 \\
\hline $\mathrm{CA}$ & -2.64 & 0.03 & -2.77 & -8.64 & $<0.001$ & -9.06 \\
\hline
\end{tabular}


a

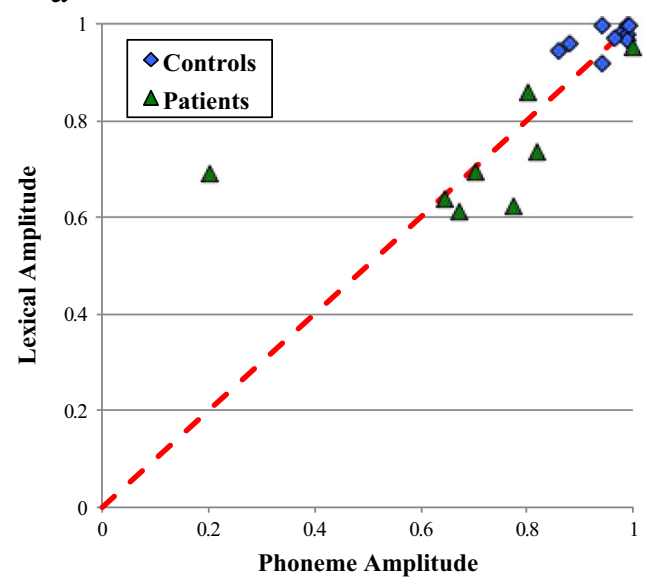

Fig. 4 Amplitude data for phoneme and lexical identification tasks. (a) Lexical identification amplitudes plotted as a function of phoneme identification amplitudes. (b) Lexical identification amplitude $z$-scores for PWAs relative to controls plotted as a function of phoneme identification amplitude $z$-scores. The red dotted line indicates $\mathrm{y}=\mathrm{x}$.

consonants (ba/pa vs. bomb/palm; Nearey \& Rochet, 1994; Stevens, 2001), it is evident that SH is severely impaired on both tasks, with amplitude suggesting slightly better performance on the phoneme (amplitude $=0.20$ ) than on the lexical (amplitude $=0.00$ ) identification task (see Supplementary Fig. S1.2 and associated caption for further discussion).

In general, PWAs who had significantly smaller amplitudes relative to controls on phoneme identification also had significantly smaller amplitudes relative to controls on the lexical identification task. Importantly, the degree of impairment (indexed using $z$-scores) was either equivalent between the two tasks or more severe on the lexical $\mathrm{b}$

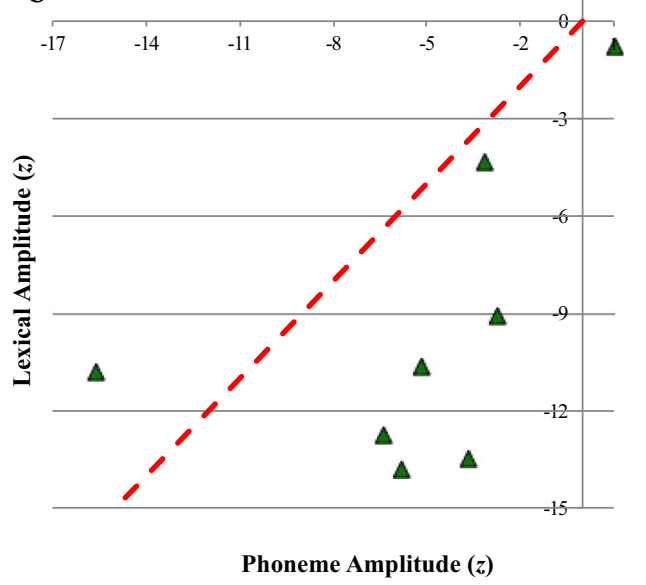

Note how amplitude $z$-scores (b) fall below the red dotted line, indicating a larger deviation from controls in the lexical identification task. The only data point that falls far from the line is that of SH (see text for discussion)

task (except SH, but see discussion above and Supplementary Fig. S1.2). Overall, the amplitude data support the hypothesis that sublexical processing necessarily precedes lexical processing.

This close alignment of amplitudes across the tasks can also be seen in Fig. 4a, which presents lexical amplitudes plotted as a function of phoneme amplitudes for PWAs and controls. Here, while the PWAs are generally lower (closer to the origin) than the controls, in both groups there is a close alignment between the amplitudes (except for $\mathrm{SH}$ ). To examine this relation further, we computed Pearson correlations with $95 \%$ confidence intervals computed by a bootstrapping procedure $(n=10,000)$. When grouping

Table 3 Slopes for phoneme and lexical identification tasks and results of Crawford and Howell's (1998) modified $t$-test comparing PWA's slopes to controls. Slopes extracted using Equation 1

\begin{tabular}{|c|c|c|c|c|c|c|}
\hline \multirow[t]{2}{*}{ PWA } & \multicolumn{3}{|l|}{ Phoneme } & \multicolumn{3}{|l|}{ Lexical } \\
\hline & Slope & $t$ & $p$ & Slope & $t$ & $p$ \\
\hline QO & 0.92 & 0.45 & 0.66 & 0.28 & -0.85 & 0.42 \\
\hline NMA & 0.86 & 0.21 & 0.84 & 0.13 & -1.40 & 0.19 \\
\hline $\mathrm{SH}$ & 0.03 & -3.12 & 0.01 & 0.15 & -1.33 & 0.22 \\
\hline MB & 0.21 & -2.38 & 0.04 & 0.51 & 0.02 & 0.98 \\
\hline EV & 0.14 & -2.68 & 0.03 & 0.22 & -1.09 & 0.30 \\
\hline NJ & 0.24 & -2.28 & 0.05 & 0.16 & -1.29 & 0.23 \\
\hline $\mathrm{HO}$ & 0.57 & -0.96 & 0.36 & 0.33 & -0.65 & 0.53 \\
\hline $\mathrm{CA}$ & 0.15 & -2.62 & 0.03 & 0.20 & -1.15 & 0.28 \\
\hline Control mean (range) & $0.80(0.40-1.00)$ & - & - & $0.50(0.26-0.98)$ & - & - \\
\hline
\end{tabular}




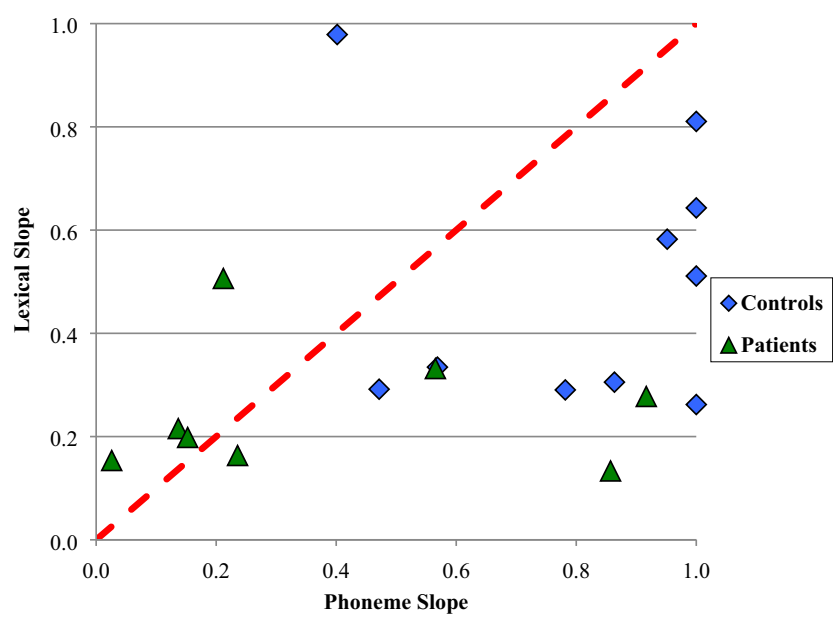

Fig. 5 Lexical identification slopes plotted as a function of phoneme identification slopes. Slopes extracted using Equation 1. The red dotted line indicates $\mathrm{y}=\mathrm{x}$

patients and controls, the correlation was strong and significant, $r(16)=0.75, p<.001,95 \%$ CI $[0.66,0.96]$, reflecting a positive relation between the phoneme and lexical amplitudes. In other words, a larger amplitude on the phoneme task, reflecting more discrete categorization of the endpoints of the VOT continuum, is related to a larger amplitude on the lexical task. One might object that the observed correlation is driven by overall aphasia severity. However, this does not appear to be the case. When examining the PWA group independently of the control group, the strength of the correlation was moderate, though not significant $(r(6)=.51, p=.19,95 \%$ CI $[-0.24,0.96])$, and when controlling for overall aphasia severity using the WAB-AQ, the size of the correlation was only minimally changed $(r=.48)$. Thus, it does not seem that the results of the correlation analysis derive from overall aphasia severity.

Analysis of slope Next, we examined the relation between the slopes of the phoneme and lexical identification functions (Table 3). It is important to note that the slope was derived from the midpoint (i.e., the category boundary), independently of the asymptotes, and represents the point-wise derivative of the function at the midpoint. In this way, it is not inherently related to the asymptote and does not scale with the amplitude.

As before, a two-way ANOVA was carried out using task (phoneme vs. lexical) as a within-subjects factor and group (PWA vs. control) as a between-subjects variable. The results showed a highly significant main effect of group, $F(1,32)=12.24, p=.001$, and a significant main effect of task $F(1,32)=7.30, p=.01$, but no interaction $(p$ $=0.34)$. For the phoneme task, six PWAs scored below the range of controls, and for the lexical task, five PWAs scored below the range of controls. Thus, as a group, the PWAs showed shallower slopes relative to controls. As for the main effect of task, the slopes were generally shallower for the lexical task. However, as previously noted, it is important to examine individual PWA's performance.

Results of Crawford and Howell's (1998) modified ttest (Table 3) identified five patients whose slopes were significantly different from the control group for phoneme identification (SH, MB, EV, NJ, CA). For lexical identification, no patients' slopes were significantly different from the control group ( $p$ 's from .19 to .98). However, when looking at the control data, the variability in the lexical slopes (range $=.26-.98$ ) was greater than the phoneme slopes (range $=.40-1.00$ ), and it would have been statistically impossible for a patient's slope to differ from controls in that task. We did not examine $z$-scores for slopes given the lack of significant difference for any PWA relative to the control group on the lexical identification task.

Figure 5 presents slope data for PWAs and controls with lexical slopes plotted as a function of phoneme slopes. As can be seen, there is no clear relation between phoneme and lexical slopes $(r(16)=.30, p=.22)$. It is clear, however, when comparing PWAs to controls that most of the patients' response data do not show a pattern like that of controls (Supplementary Figs. S1.3 and S1.4; see also Fig. S1.1, showing the response data for individual lexical stimuli). Whereas the controls consistently displayed a sigmoidal curve, a hallmark of accurate and discrete speech perception/categorization, many of the PWAs had very shallow slopes and failed to show clear asymptotes. As a result, the four-parameter logistic function may have not adequately described the observed data, as the same participant could have been equally well fit, as indexed by $R^{2}$, by a function with asymptotes near 0 and 1 but a very shallow slope, or by one with asymptotes closer to .5 (and perhaps a steep slope).

To investigate this further, we conducted an additional analysis using the pooled data (reported in Supplement S3). This constrained the analysis to a two-parameter logistic function using binomial mixed-effects modeling. Individual slopes were extracted from the binomial mixed-effects model, and statistical analyses confirmed that all but one PWAs (HO) had shallower slopes than controls on both measures.

\section{Eye-movement data}

We next examined the competitor fixations as a more implicit measure of the dynamics of the lexical or phoneme identification. Eye-movement recording was time-locked to $300 \mathrm{~ms}$ after trial onset. This includes $200 \mathrm{~ms}$ (to 


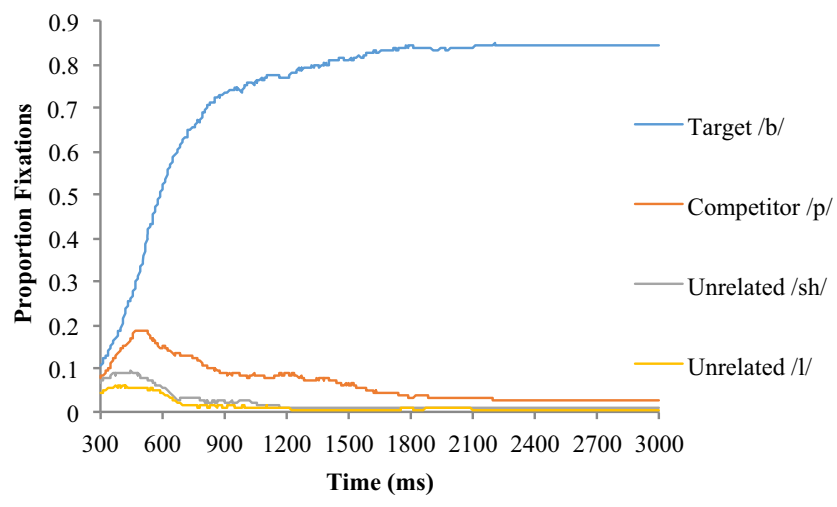

Fig. 6 Control looks to target, competitor, and unrelated stimuli in the lexical identification task at rStep -3

account for the amount of time required to initiate an eye movement), and an additional $100 \mathrm{~ms}$ as the auditory stimuli were preceded by $100 \mathrm{~ms}$ of silence. Eye-movement recording ended when the participant made their response. Similar to prior work (e.g., McMurray et al., 2002), if the trial ended before a fixed cut off $(3,000 \mathrm{~ms})$ the final fixation was extended to that cut off. We computed the proportion of trials on which the participant was fixating each class of responses every $4 \mathrm{~ms}$.

Our initial goal was to examine the sensitivity of competitor looking to within-category differences in VOT. That is, given that the listener knows that an auditory stimulus is $/ b a /$ (or $/ p a)$, how strongly are they considering the competitor as a function of VOT? Our initial plan was to utilize the theoretical framework put forward by McMurray et al. (2014) to localize the source of each patient's deficit based on their individual profiles of sensitivity to within-category differences in VOT. To accomplish this, we needed to examine sensitivity to specifically within-category differences in VOT (e.g., 5 vs. $10 \mathrm{~ms}$, both of which result in $\mathrm{a} / \mathrm{b} /$ decision). To ensure this, we took two steps, as in McMurray et al. (2008).

First, to account for variability in participant's boundaries, we calculated VOT steps relative to the category boundary (rStep) for each participant. Here, an rStep of 0 indicates a VOT precisely at the category boundary (see McMurray et al., 2014), negative VOTs indicate a /b/ and positive a $/ \mathrm{p} /$. Second, we only analyzed fixation data for rSteps on the voiced side (negative) when the /b/-initial stimulus was actually selected; likewise, on the voiceless side (positive rSteps) fixations were only analyzed when the /p/-initial stimulus was selected. For phoneme identification, removal of trials where the competitor was ultimately chosen or where the trial timed out led to the exclusion of $3.4 \%$ of the data for controls (range $=.93-$ $8.2 \%$ ) and between $5.6 \%$ and $21.3 \%$ for PWAs; for lexical identification, this constituted $3.8 \%$ of the data for controls (range $=1.6-6.6 \%)$ and between $4.7 \%$ and $18.5 \%$ for PWAs.

We then determined the proportion of looks to the competitor as a function of VOT (rStep) at each 4-ms time bin. For example, Fig. 6 shows fixation data from the lexical identification task on the voiced side of the continuum (rStep -3) for control participants. As can be seen, there was rapid elimination of the unrelated stimuli (/sh/ and /1/ initial) compared to a more gradual elimination of the competitor stimulus $(/ \mathrm{p} /$ initial) as the target stimulus $(/ \mathrm{b} / \mathrm{ini}-$ tial) began to dominate fixations.

Finally, for statistical analyses, the dependent variable was the mean proportion of looks to the competitor across trials, the area under the curve (AUC). This would be, for example, the area under the orange line in Fig. 6. Larger AUCs thus correspond to increased competitor looking. The AUC was calculated across the entire trial (i.e., 300$3,000 \mathrm{~ms}$, with the final fixation extended on trials where a response was made before $3,000 \mathrm{~ms}$ ). We also explored other time windows $(300-1,000 \mathrm{~ms}, 300-2,000 \mathrm{~ms}$, $1,000-3,000 \mathrm{~ms}$ and $1,800-3,000 \mathrm{~ms}$ ). These analyses were not substantially different from those for the entire trial, so the entire trial was used for subsequent analyses. ${ }^{7}$

Initial attempts looked at AUC as a function of VOT within listeners as has been typically done at the group level (e.g., McMurray et al., 2014). However, attempts to estimate this gradiency at a single-subject level showed highly variable functions that did not correspond to any expected pattern, even in the control group. This suggests we may have lacked sufficient power at the single-subject level. Consequently, a comparison of PWAs to controls would not be interpretable, and thus these data are not reported here.

As a result, we computed the average AUCs across rSteps (collapsed across word pairs for lexical identification, excluding poorly fit stimuli) for each side of the category boundary, and subsequently averaged across the $/ b /$ and $/ p /$ sides. Even though this cannot speak to sensitivity to VOT, this offers a precise measure of how much competition the listener is undergoing along the way to ultimately selecting the target. That is, even on trials on which the participant was ultimately "correct," this approach asks whether they showed more or less competition from the competitor stimulus. The AUC for PWAs and controls is presented in Table 4.

As with the identification data, a two-way ANOVA was carried out using task (phoneme vs. lexical) as a withinsubjects factor and group (PWA vs. control) as a between-

\footnotetext{
${ }^{7}$ All PWA who were significantly different from controls in the 300-3,000-ms time window were also different from controls in at least two other time windows. Further, no PWA was significantly different from controls in the other time windows who was not different from controls in the selected time window.
} 


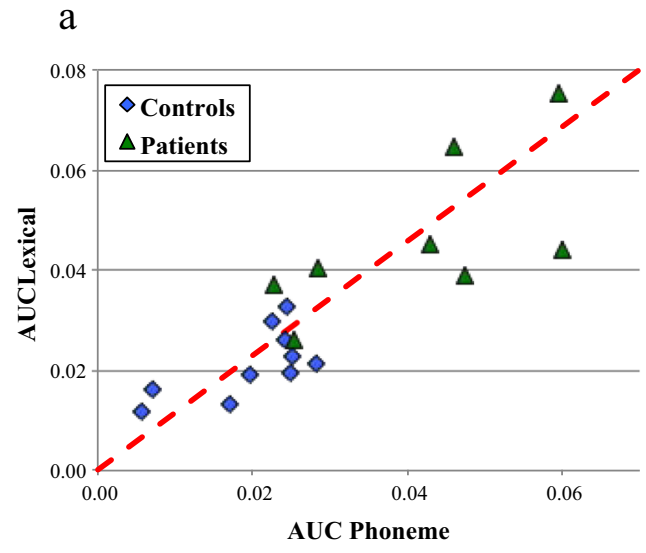

Fig. 7 AUC data for phoneme and lexical identification tasks. (a) Lexical identification AUC plotted as a function of phoneme identification AUC for PWA and controls. (b) Lexical identification AUC $z$-scores for PWA. relative to controls plotted as a function of phoneme identification $\mathrm{AUC} z-$ scores. The red dotted line indicates $\mathrm{y}=\mathrm{x}$. Note how most AUC $z$-scores

subjects variable. The results showed a highly significant main effect of group, $F(1,32)=31.44, p<.001$, but no main effect of task $(p=.77)$ and no interaction $(p=.84)$. For the phoneme task, five PWAs had AUCs that were outside of the control range, and for the lexical task, all PWAs had AUCs that were outside of the control range (when rounded to three decimals). Again, though, it is more critical to examine individual patient scores relative to the control group.

Crawford and Howell's (1998) modified $t$-tests were used to determine whether each patient's AUC was significantly different from the control group. The modified $t$ test revealed five PWAs (NMA, SH, MB, EV, CA) who had significantly larger AUCs (more competition) for the phoneme task compared to controls (Table 4). For lexical b

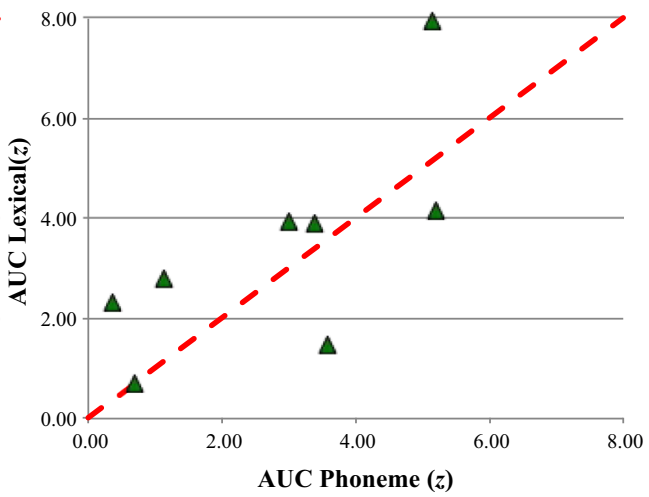

(b) fall near or above the red dotted line, indicating a similar or larger proportion of competitor fixations for lexical identification relative to phoneme identification. The two data points that fall below the line represent $\mathrm{MB}$ and $\mathrm{CA}$ (see text for discussion)

identification, four of these PWAs (NMA, SH, EV, CA) as well as two additional PWAs (NJ, QO) had significantly larger AUCs than controls. However, the AUC for MB on the lexical task was not significantly different from controls, though it was just outside the high end of the normal control range. As before, it is unclear to what extent exclusion of poorly fit word pairs masks the nature of the impairment (two word pairs were excluded from analyses for MB because they were poorly fit by Eq. 1), and so we note that when including all word pairs in the analysis, MB's AUC was significantly larger than that for controls (see Supplementary Table S2.4 for data including poorly fit word pairs).

The degree of impairment, as indexed by $z$-scores (Table 4, Fig. 7b), was roughly equivalent or larger for

Table 4 PWA and control AUC data for competitor fixations when target selected for phoneme and lexical identification tasks, results of Crawford and Howell's (1998) modified $t$-test comparing PWA's AUCs to controls and $z$-scores relative to controls

\begin{tabular}{|c|c|c|c|c|c|c|c|c|}
\hline \multirow[t]{2}{*}{ PWA } & \multicolumn{4}{|l|}{ Phoneme } & \multicolumn{4}{|l|}{ Lexical } \\
\hline & AUC & $t$ & $p$ & $z$ & AUC & $t$ & $p$ & $z$ \\
\hline QO & 0.02 & 0.35 & 0.74 & 0.36 & 0.04 & 2.23 & 0.05 & 2.34 \\
\hline NMA & 0.06 & 4.89 & $<0.001$ & 5.13 & 0.08 & 7.57 & $<0.001$ & 7.94 \\
\hline $\mathrm{SH}$ & 0.05 & 3.20 & 0.01 & 3.36 & 0.05 & 3.73 & $<0.01$ & 3.91 \\
\hline MB & 0.05 & 3.40 & 0.01 & 3.56 & 0.03 & 1.43 & 0.19 & 1.50 \\
\hline EV & 0.04 & 2.85 & 0.02 & 2.99 & 0.05 & 3.76 & $<0.01$ & 3.95 \\
\hline NJ & 0.03 & 1.06 & 0.32 & 1.11 & 0.04 & 2.67 & 0.03 & 2.80 \\
\hline $\mathrm{HO}$ & 0.03 & 0.66 & 0.53 & 0.69 & 0.03 & 0.69 & 0.51 & 0.72 \\
\hline $\mathrm{CA}$ & 0.06 & 4.95 & $<0.001$ & 5.19 & 0.05 & 3.97 & $<0.01$ & 4.16 \\
\hline Control mean (range) & $0.02(0.01-0.03)$ & - & - & - & $0.02(0.01-0.03)$ & - & - & - \\
\hline
\end{tabular}


the lexical task than the phoneme task for six of the PWAs (QO, NMA, SH, EV, NJ, HO); MB's and CA's were smaller for the lexical than the phoneme task (MB: $z$ phoneme $=$ $3.56, z$ lexical $=1.50$ (2.59 including all word pairs); CA: $z$ phoneme $=5.19, z$ lexical $=4.16$ (3.40 including all word pairs)) . Although MB and CA were slightly more impaired on the sublexical task, both MB's and CA's AUCs for the lexical task were still outside of the control range, indicating non-normal performance. This indicates that even though the phoneme AUCs were more different from controls than the lexical AUCs for these two PWAs, there was still a trend for lexical being impaired.

Figure 7a presents AUC data for the lexical identification task plotted as a function of the phoneme identification task AUCs. There is a clear relation between the phoneme and lexical AUCs. To examine this relation further, we computed Pearson correlations, as before. Across patients and controls, the correlation was strong and significant, $r(16)=.85, p<.001,95 \%$ CI $[.67, .94]$, suggesting a positive relation between the phoneme and lexical AUCs. When examining the PWA group independently of the control group, the correlation was strong and significant $(r(6)=.70, p=.05,95 \%$ CI $[0.16$, $0.96]$ ), and when controlling for overall aphasia severity using the WAB-AQ, the size of the correlation was essentially the same $(r=.69)$, suggesting that aphasia severity is not a confound in this analysis.

\section{General discussion}

This study examined the relation between speech processing at the sublexical and lexical levels for individuals with aphasia by examining the effects of a VOT manipulation on phoneme and word identification. This study marks the first attempt to compare the influence of VOT manipulation on sublexical and lexical processing tasks in the same PWAs. We used these data to ask whether an intermediate stage exists between acoustic and lexical processing, where acoustic information is mapped onto abstract, speechspecific sublexical representations prior to lexical access. We hypothesized that if lexical processing depends on sublexical processing, then sublexical processing deficits should cascade to affect the lexical level, and as a result, PWAs should be equally or more impaired on the lexical compared to the sublexical processing task. In general, the findings of the current study support this hypothesis. All group analyses revealed significantly worse performance for the PWA relative to the control group and no interaction between group and the difference between performance on the lexical and sublexical tasks. Moreover, all PWAs were more impaired on the word-identification task than the phoneme identification task for at least one dependent measure (except $\mathrm{HO}$, who was within the control range on all measures), and the overall pattern that emerged was one of equally or more impaired performance on the lexical task. This is consistent with a model in which lexical level processing is dependent on sublexical processing.

While the primary support for our hypothesis derives from the differences between phoneme and lexical tasks across participants, we also generally observed moderate or strong correlations between all dependent measures on the sublexical (phoneme) and lexical (word) identification tasks. These correlations offer two insights. First, they emphasize that when tasks are closely matched in terms of the perceptual discriminability of targets and distractors, performance on the two tasks shows a clear relation. This contrasts with previous work in which sublexical and lexical processing tasks were not closely matched (e.g., Blumstein et al., 1977a). Second, these correlations allow us to capture the pattern of performance across individuals. This is fundamental to use of brain damaged patients as it speaks to what patterns are possible. For example, the presence of one or more PWAs with impaired sublexical performance, but good lexical performance (for example) could have led to low correlations, and would have challenged our hypothesis. ${ }^{8}$ The high correlations thus reflect the fact that when sublexical processing was impaired (or spared), lexical processing was also relatively impaired (or spared). This characterizes the broad pattern of data. However, it is the finding of more impaired performance on the lexical than on the sublexical task at the individual patient level that is critically important in supporting the hypothesis that lexical processing is dependent upon sublexical processing.

For some participants on some dependent measures (i.e., amplitude: SH; AUC: MB, CA), the degree of deficit, indexed using $z$-scores, was larger for the phoneme than the word-identification task. In each of these relatively few cases, lexical processing was also impaired relative to the control group, but sublexical processing was more impaired. And, in each of these cases, alternative explanations exist for the relatively worse performance on the phoneme identification task. For SH's amplitude, when examining the most closely matched stimuli (ba/pa and bomb/palm), the amplitude was larger for the phoneme

\footnotetext{
${ }^{8}$ We note though that non-significant correlations between the dependent measures for the sublexical and lexical tasks could also be consistent with the hypothesis that sublexical processing is required for lexical processing. This could occur if performance on the lexical processing task could be impaired for reasons other than impaired sublexical processing, such as deficits at the lexical-phonological or lexical-semantic level. Thus, a null correlation would only be a marker to look more closely at the distribution of patients, not evidence for either hypothesis in isolation.
} 
task, indicating more normal performance. And, for the AUC for MB and CA, exclusion of poorly fit word pairs may have skewed the results, though for MB the degree of impairment was larger for the sublexical task relative to the lexical task even when including all word pairs. We would note, though, that for MB and CA, the dependent measures derived from the identification responses (amplitude, slopes extracted using Equation S3.1) were more deviant from controls for the lexical than the sublexical processing task.

\section{Inferring PWA deficits from the identification and AUC data}

In general, on the identification measures many of the PWAs exhibited little or no difference between the asymptotes, a much shallower slope than the control group, and an increase in competitor fixations. What can account for this? To address this question in detail, it is helpful to consider the processes that support performance in speech categorization tasks, particularly identification tasks like used here. In the classic interpretation of these tasks (e.g., Repp, 1984), individuals must first encode the low-level acoustic cues of the perceived stimulus - that is, they must have adequate hearing abilities to perceive the stimuli. Next, these acoustic cues must be mapped to a category (e.g., /ba/ or $/ p a /)$. Finally, the participant must make a response (e.g., selecting the target in either a two- or four-alternative forced choice paradigm). This loose framework can help interpret our patients' small amplitudes, shallow slopes, and large AUCs.

First, decreased amplitudes indicate that, even when the VOT is unambiguous, participants did not respond at $100 \%$ $/ b a /$ (or $/ p a /)$. One potential explanation for this impairment is noisy encoding of the auditory cues (e.g., VOT). However, this explanation is unlikely to explain the difficulties we observed specifically at the end-points (the decreased amplitudes). For example, noisy encoding of a VOT of $0 \mathrm{~ms}$ might lead to encoding the auditory input on a given trial as a VOT of $10 \mathrm{~ms}$. However, this would still fall on the voiced side of the category boundary, so it would not change the ultimate response. Thus, if the only problem were noisy encoding of acoustic cues, performance at the endpoint would still be close to $100 \%$ (McMurray et al., 2018). In contrast, this might have a large effect if the actual VOT were $10 \mathrm{~ms}$ - here noise could lead the subject to hear the VOT as 20, and respond $/ \mathrm{p} /$ on some trials. Thus, this predicts a difference in slope, but not amplitude.

Instead, decreased amplitudes are more likely to be caused by a failure to resolve competition, either at the level of category representations or at the response stage. That is, even for an unambiguous VOT of $0 \mathrm{~ms}$, the competing category (or response) $/ \mathrm{p} /$ is likely to still be active - it shares place of articulation and manner, and it is relevant to the task. In the lexical case, there is even more cause for this: when beach is heard, the -each is consistent with the competing word (/peach/). Under typical circumstances, this activation for the competitor would rapidly be suppressed in the moment for the listener to respond accurately. However, if the PWA struggled to resolve this competition, they may have inconsistent responses, even at the endpoints of the continuum. ${ }^{9}$ Moreover, given that this could occur at the level of the phoneme, word, or response, it is unlikely to be related to the VOT, but would manifest itself as poor performance across the continuum.

In line with this hypothesis, many of the PWAs had larger AUCs as compared to the control group, indicating that they were more likely to fixate the competitor stimulus and, accordingly, less likely to successfully eliminate the competitor from consideration. In other words, larger AUCs were likely driven by a failure (or decreased ability) to resolve competition between the target stimulus and the competitor stimulus. Mirman et al. (2011) also suggested a faulty response selection/competition resolution mechanism in individuals with Broca's and Wernicke's aphasia in a combined visual world paradigm (eye-tracking) and computational modeling study. In this study, distractors were either rhyme or cohort competitors. They found that individuals with Broca's aphasia were more likely to select rhyme competitors, whereas individuals with Wernicke's aphasia were more likely to select cohort competitors. Computational modeling of the observed fixation data within the TRACE framework suggested that both patterns could be explained by a failure to resolve lexical competition. This is in line with theoretical and empirical work suggesting the necessity of resolving competition for good language learning and processing (McMurray et al., 2009, 2018).

Finally, the analysis of the identification slopes shows that the PWA did not exhibit discrete categorization of the stimuli. This aspect of the listeners' performance could be attributed to noisy mapping of acoustic cues to category representations at the sublexical level. If the PWA had difficulty mapping acoustic cues to categories, as the VOT moved away from the endpoint, PWA would be more likely to choose the competitor stimulus, leading to a shallower slope than what is typically observed in categorical perception studies. In fact, this is what is reflected in the current data for several of the PWA (e.g., EV, SH, NJ, CA, Supplementary Fig. S1.4), leading us to conclude

\footnotetext{
${ }^{9}$ The current data are not sufficient to parse out the level at which competition resolution is faulty, and is an area for future research.
} 
that some of the PWAs were relying quite heavily on the continuous acoustic information and failing to robustly map this information onto a categorical representation. The ability to map this continuous information onto discrete, categorical representations may be one distinguishing feature between normal and impaired speech perception. As such, the participants with the most shallow identification slopes were also those who appeared to be the most impaired relative to the control group.

Taken together, there are likely two separate deficits that can explain the impaired performance of PWA in the current study. First, shallower identification slopes suggest noisy mapping of acoustic cues to category representations at the sublexical level. Second, decreased amplitudes and larger AUCs suggest a deficit in the competition resolution mechanism (either at the level of category representations or at the response stage) that is required for successful identification of incoming speech stimuli. These deficits are not mutually exclusive, and some PWAs may have noisy mapping of acoustic cues to category representations, combined with decreased competition resolution abilities (e.g., SH, MB, EV, and CA, who were all significantly impaired relative to controls on amplitude, slope, and AUC measures).

\section{Using sublexical tasks to assess sublexical processing}

Some researchers have argued that tasks that utilize sublexical units of representation (e.g., phonemes) do not accurately reflect sublexical processing. Instead, researchers argue that these tasks may be tapping into cognitive processes beyond those involved in normal speech perception. For example, Hickok and Poeppel (2007) assert that executive control and/or working memory processes may be required to perform sublexical tasks, and that the use of sublexical tasks may not measure the sublexical process they are assumed to measure. As another example, Gow (2012) and Norris et al. (2000) have argued that performance on tasks requiring identification of sublexical units of representation does not reflect purely sublexical processing abilities. Instead, these researchers argued that identification of phonemes in these tasks is made at a post-lexical decision-making stage (e.g., the phoneme decision level; Merge model, Norris et al., 2000).

We do not deny these important points, and our goal was not specifically to inform our understanding of the use of sublexical tasks to assess sublexical processing. However, our findings do speak to this issue. Specifically, if phoneme identification requires cognitive processes beyond those required in normal speech perception, or if it is a post-lexical form of processing, then we should have observed that some PWAs perform more poorly on the phoneme than lexical identification task due to deficits in executive function, working memory, and/or post-lexical decision-making. However, we did not observe this pattern in any of the PWAs. Instead we observed a strong correlation between measures of sublexical and lexical processing obtained from the phoneme and lexical identification tasks. When dissociations between performance on the sublexical and lexical tasks were observed, it was because some PWAs performed worse on the lexical than sublexical task. This supports the contention that sublexical and lexical processing are distinct processes, but that it is lexical level processing that follows downstream from sublexical level processing. Together, this supports the claim that performance on tasks using sublexical (i.e., phonemic) stimuli reflect sublexical processing abilities.

\section{Summary and conclusions}

Speech perception involves the translation of acoustic cues into units that allow access to the meaning of what is being said. Many models of speech perception have assumed that abstract, speech-specific sublexical processing is a necessary prerequisite for lexical processing (e.g., Cole \& Scott, 1974; Hickok \& Poeppel, 2007; McClelland \& Elman, 1986; Luce, Goldinger, Auer, \& Vitevitch, 2000; Luce \& Pisoni, 1998; Norris, 1994; Norris, McQueen, \& Cutler, 2000; Oden \& Massaro, 1978). However, evidence from PWAs suggests that sublexical and lexical processing may be dissociable. In part due to this evidence, researchers have proposed models of speech perception where auditory information can map directly to lexical levels (Goldinger, 1998; Hickok \& Poeppel, 2000; Johnson, 1997; Pierrehumbert, 2001).

In the current study, we obtained evidence for the obligatory sublexical processing approach. When sublexical processing was impaired, lexical processing was equally or more impaired on at least one of the dependent measures. These data provide converging evidence with the findings from Dial and Martin (2017) using a different task, a more finegrained behavioral measure and eye-movement data, which provides a measure of online speech processing.

Acknowledgements We wish to acknowledge the contribution of the participants, particularly those with aphasia, to the current study. We would like to thank the members of the Brain and Language Lab at Rice University and the MACLab at University of Iowa for assistance. Finally, we would like to thank the reviewers for their thoughtful comments and suggestions. This work was supported by the Gertrude Maurin fund (HRD) and the T.L.L. Temple Foundation Neuroplasticity Lab (RM) at Rice University and NIH grant DC 008089 (BM).

Data for the experiments reported here are available at: https://osf.io/ $9 \mathrm{z} 8 \mathrm{x} 2$. 


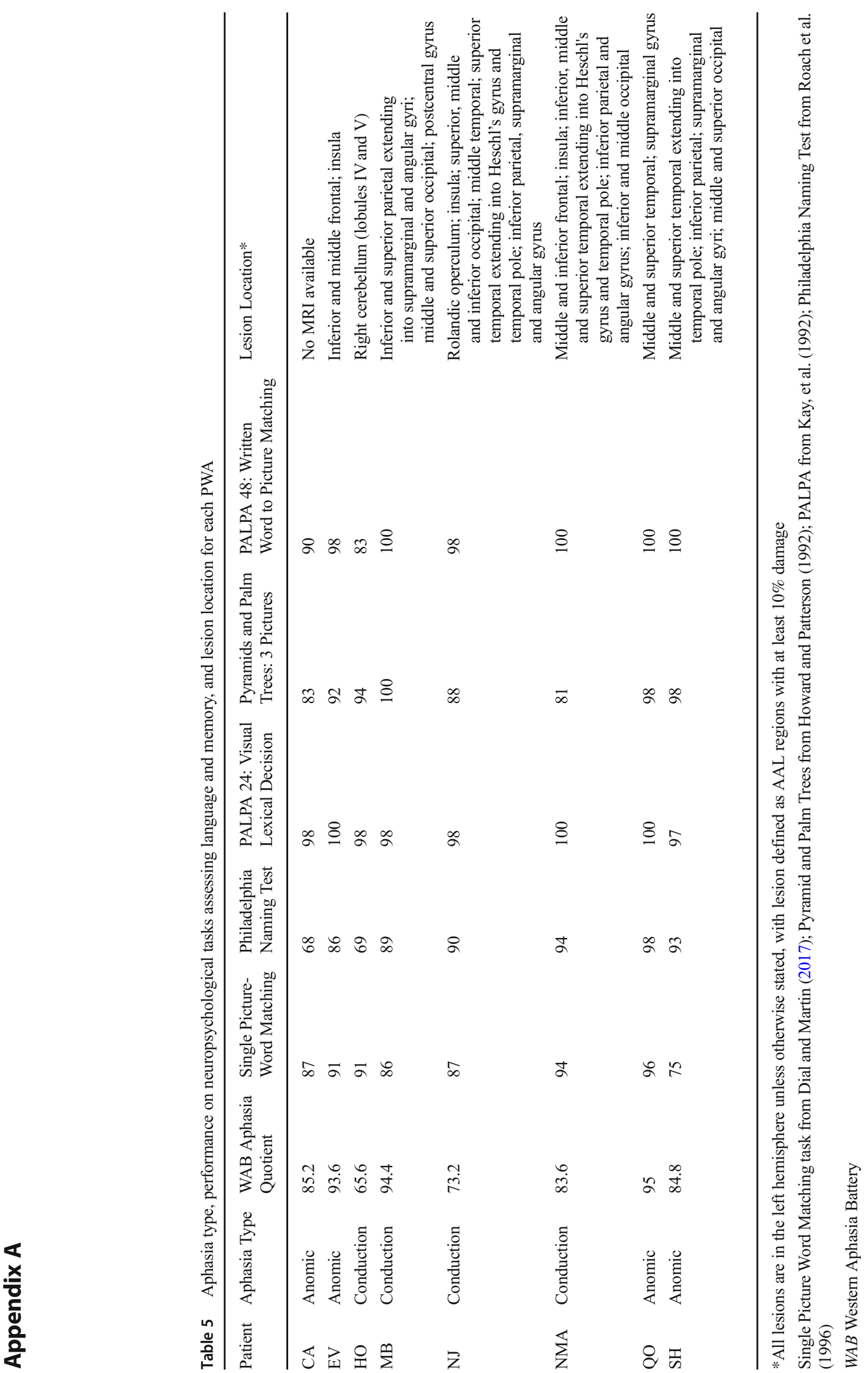




\section{Appendix B}

Table $6 \quad \mathrm{R}^{2}$ of fit for PWA for the phoneme and lexical identification tasks

\begin{tabular}{llll}
\hline PWA & Phoneme & Lexical: All Word Pairs & Lexical: Excluding Poorly Fit Word Pairs \\
\hline QO & .987 & .995 & .995 \\
NMA & .975 & .980 & .980 \\
SH & .787 & .992 & .997 \\
MB & .955 & .983 & .980 \\
EV & .991 & .980 & .983 \\
NJ & .973 & .997 & .997 \\
HO & .999 & .996 & .996 \\
CA & .951 & .981 & .984 \\
Control Mean & .994 & .999 & .999 \\
\hline
\end{tabular}

\section{References}

Allopena, P., Magnuson, J., \& Tanenhaus, M. (1998). Tracking the time course of spoken word recognition using eye movements: Evidence for continuous mapping models. Journal of Memory and Language, 38, 419-439. doi:https://doi.org/10.1006/jmla.1997.2558

Basso, A., Casati, G., \& Vignolo, L. (1977). Phonemic identification defect in aphasia. Cortex, 13, 85-95. doi:https://doi.org/10.1016/ s0010-9452(77)80057-9

Blumstein, S. E., Baker, E., \& Goodglass, H. (1977a). Phonological factors in auditory comprehension in aphasia. Neuropsychologia, 15(1), 19-30. doi:https://doi.org/10.1016/0028-3932(77)90111-7

Blumstein, S., Burton, M., Baum, S., Waldstein, R., \& Katz, D. (1994). The role of lexical status on the phonetic categorization of speech in aphasia. Brain and Language, 46, 181-197. doi: https://doi.org/10. 1006/brln.1994.1011

Blumstein, S., Cooper, W., Zurif, E. \& Caramazza, A. (1977b). The perception and production of voice-onset time in aphasia. Neuropsychologia, 15, 371-383. doi:https://doi.org/10.1016/00283932(77)90089-6

Chen, Q., \& Mirman, D. (2012). Competition and cooperation among similar representations: Toward a unified account of facilitative and inhibitory effects of lexical neighbors. Psychological Review, 119, 417-430. doi: https://doi.org/10.1037/a0027175

Cole, R. A., \& Scott, B. (1974). Toward a theory of speech perception. Psychological Review, 81(4), 348. doi:https://doi.org/10.1037/ h0036656

Crawford, J.R., Howell, D.C., 1998. Comparing an individual's test score against norms derived from small samples. The Clinical Neuropsychologist, 12, 482-486.

Dahan, D., Magnuson, J.S., Tanenhaus, M.K. \& Hogan, E. (2001) Subcategorical mismatches and the time course of lexical access: Evidence for lexical competition. Language and Cognitive Processes, 16, 507-534. doi:https://doi.org/10.1080/ 01690960143000074

Dalton, D., Cruickshanks, K., Klein, B., Klein, R., Wiley, T., Nondahl, D., 2003. The impact of hearing loss on quality of life in older adults. The Gerontol. 43, 661-668. https://doi.org/10.1093/geront/43.5. 661.

Dial, H.R., \& Martin, R.C. (2017). Evaluating the relationship between sublexical and lexical processing in speech perception: Evidence from aphasia. Neuropsychologia, 96, 192-212. doi:https://doi.org/ 10.1016/j.neuropsychologia.2017.01.009
Diehl, R. L., Lotto, A. J., \& Holt, L. L. (2004). Speech perception. Annual Review of Psychology, 55, 149-179. doi:https://doi.org/10.1146/ annurev.psych.55.090902.142028

Goldinger, S. D. (1998). Echoes of echoes? An episodic theory of lexical access. Psychological Review, 105(2), 251-279. doi:https://doi.org/ 10.1037/0033-295x.105.2.251

Gow, David. (2012). The cortical organization of lexical knowledge: A dual lexicon model of spoken language processing. Brain and Language, 121, 273-288. doi:https://doi.org/10.1016/j.bandl/2012. 03.005

Hickok, G. (2014). The architecture of speech production and the role of the phoneme in speech processing. Language, Cognition and Neuroscience, 29, 2-20. doi:https://doi.org/10.1080/01690965. 2013.834370

Hickok, G., \& Poeppel, D. (2000). Towards a functional neuroanatomy of speech perception. Trends in Cognitive Sciences, 4(4), 131-138. doi: https://doi.org/10.1016/s1364-6613(00)01463-7

Hickok, G., \& Poeppel, D. (2004). Dorsal and ventral streams: A framework for understanding aspects of the functional anatomy of language. Cognition, 92, 67-99. doi:https://doi.org/10.1016/j.cognition. 2003.11.001

Hickok, G., \& Poeppel, D. (2007). The cortical organization of speech processing. Nature Reviews Neuroscience, 8, 292-402. doi:https:// doi.org/10.1038/nrn2113

Huettig, F., \& Atlmann, G. (2005). Word meaning and the control of eye fixation: Semantic competitor effects and the visual world paradigm. Cognition, 96, B23-B32. doi: https://doi.org/10.1016/j.cognition. 2004.10.003

International Organization for Standardization, 2000. AcousticsStatistical Distribution of Hearing Thresholds as a Function of Age. ISO7029. Geneva: ISO.

Johnson, K. (1997). Speech perception without speaker normalization: An exemplar model. In Johnson \& Mullenix (Eds.), Talker variability in speech processing (145-165). San Diego: Academic Press.

Liberman, A. M., Harris, K. S., Hoffman, H. S., \& Griffith, B. C. (1957). The discrimination of speech sounds within and across phoneme boundaries. Journal of Experimental Psychology, 54(5), 358-368. doi:https://doi.org/10.1037/h0044417

Luce, P., Goldinger, S., Auer, E., \& Vitevitch, M. (2000). Phonetic priming, neighborhood activation and PARSYN. Perception and Psychophysics, 62, 615-625. doi:https://doi.org/10.3758/ bf03212113 
Luce, P., \& Pisoni, D. (1998). Recognizing spoken words: The neighborhood activation model. Ear and Hearing, 19, 1-36. doi:https://doi. org/10.1097/00003446-199802000-00001

MATLAB and Statistics Toolbox Release (2015). The MathWorks, Inc., Natick, Massachusetts, United States.

McClelland, J., \& Elman, J. (1986). The TRACE model of speech perception. Cognitive Psychology, 18, 1-86. doi:https://doi.org/10. 1016/0010-0285(86)90015-0

McMurray, B. (2017a). EyelinkAnal. Available from: http://osf.io/c35tg

McMurray, B. (2017b) Nonlinear curvefitting for Psycholinguistics. Version XX. Available from: https://osf.io/4atgv/

McMurray, B., Aslin, R., Tanenhaus, M., Spivey, M., \& Subik, D. (2008). Gradient sensitivity to within-category variation in words and syllables. Journal of Experimental Psychology: Human Perception and Performance, 34(6), 1609-1631. doi: https://doi.org/10.1037/ a0011747

McMurray, B., Aslin, R. N., \& Toscano, J. C. (2009). Statistical learning of phonetic categories: insights from a computational approach. Developmental Science, 12(3), 369-378. doi:https://doi.org/10. $1111 /$ j.1467-7687.2009.00822.x

McMurray, B., Danelz, A., Rigler, H. \& Seedorff, M. (2018). Speech categorization develops slowly through adolescence. Developmental Psychology, 54, 1472-1491. doi: https://doi.org/10. 1037/dev0000542

McMurray, B., Munson, C., \& Tomblin, J.B. (2014). Individual differences in language ability are related to variation in word recognition, not speech perception: Evidence from eye movements. Journal of Speech, Language and Hearing Research, 57, 1344-1362. doi: https://doi.org/10.1044/2014_JSLHR-L-13-0196.doi:10.1016/j. cogpsych.2009.06.003

McMurray, B., Samuelson, V. M., Lee, S. H., \& Tomblin, J. B.(2010). Individual differences in online spoken word recognition: Implications for SLI. Cognitive Psychology, 60, 1-39.

McMurray, B., Tanenhaus, M., \& Aslin, R. (2002). Gradient effects of within-category phonetic variation on lexical access. Cognition, 86(2), B33-B42. doi: https://doi.org/10.1016/S0010-0277(02) 00157-9

Miceli, G., Gainotti, G., Caltagirone, C., \& Masullo, C. (1980). Some aspects of phonological impairment in aphasia. Brain and
Language, 11, 159-169. doi:https://doi.org/10.1016/0093-934x(80) 90117-0

Miller, J.L. \& Volaitis, L.E. (1989). Effect of speaking rate on the perceptual structure of a phonetic category. Perception \& Psychophysics, 46(6), 505-512. https://doi.org/10.3758/BF03208147

Mirman, D., Yee, E., Blumstein, S., \& Magnuson, J. (2011). Theories of spoken word recognition deficits in aphasia: Evidence from eyetracking and computational modeling. Brain and Language, 117, 53-68.

Nearey, T.M. \& Rochet, B.L. (1994). Effects of place of articulation and vowel context on VOT production and perception for French and English stops. Journal of the International Phonetic Association, 24(1), 1-18. doi:https://doi.org/10.1017/S0025100300004965

Norris, D. (1994). Shortlist: A connectionist model of continuous speech recognition. Cognition, 52, 189-234. doi:https://doi.org/10.1016/ 0010-0277(94)90043-4

Norris, D., McQueen, J., \& Cutler, A. (2000). Merging information in speech recognition: Feedback is never necessary. Behavioral and Brain Sciences, 23, 299-370. doi:https://doi.org/10.1017/ s0140525x00003241

Oden, G. \& Massaro, D.W. (1978). Integration of featural information in speech perception. Psychological Review, 85(3), 172-191. doi: https://doi.org/10.1037/0033-295X.85.3.172

Pierrehumbert, J. B. (2001). Exemplar dynamics: Word frequency, lenition and contrast. In Bybee \& Hopper (Eds.), Frequency effects and emergent grammar (45-64). Amsterdam: John Benjamins.

Repp, B. (1984). Categorical perception: Issues, methods, findings. In: N. Lass (Ed.), Speech and language: Advances in basic research and practice (243-335). Orlando, Florida: Academic Press, Inc.

Salverda, A.P., Dahan, D. \& McQueen, J. (2003). The role of prosodic boundaries in the resolution of lexical embedding in speech comprehension. Cognition, 90(1), 51-89. doi:https://doi.org/10.1016/ S0010-0277(03)00139-2

Publisher's note Springer Nature remains neutral with regard to jurisdictional claims in published maps and institutional affiliations. 